University of Wollongong

Research Online

Faculty of Engineering - Papers (Archive)

Faculty of Engineering and Information

Sciences

2002

\title{
Effect of various mechanical deformation techniques on pinning force densities in $\mathrm{Ag} / \mathrm{Bi}-2223$ tapes
}

W M. Chen

University of Wollongong

Hua-Kun Liu

University of Wollongong, hua@uow.edu.au

X K. Fu

University of Wollongong

Y C. Guo

University of Wollongong

Dongqi Shi

University of Wollongong, dongqi@uow.edu.au

See next page for additional authors

Follow this and additional works at: https://ro.uow.edu.au/engpapers

Part of the Engineering Commons

https://ro.uow.edu.au/engpapers/541

\section{Recommended Citation}

Chen, W M.; Liu, Hua-Kun; Fu, X K.; Guo, Y C.; Shi, Dongqi; and Dou, S. X.: Effect of various mechanical deformation techniques on pinning force densities in Ag/Bi-2223 tapes 2002.

https://ro.uow.edu.au/engpapers/541

Research Online is the open access institutional repository for the University of Wollongong. For further information contact the UOW Library: research-pubs@uow.edu.au 


\section{Authors}

W M. Chen, Hua-Kun Liu, X K. Fu, Y C. Guo, Dongqi Shi, and S. X. Dou 


\title{
EFFECT OF VARIOUS MECHANICAL DEFORMATION TECHNIQUES ON PINNING FORCE DENSITIES IN AG/BI-2223 TAPES
}

\author{
W. M. Chen, H. K. Liu, X. K. Fu, Y. C. Guo, D. Q. Shi and S. X. Dou \\ Institute for Superconducting and Electronic Materials, University of \\ Wollongong, Wollongong, New South Wales 2522, Australia.
}

\begin{abstract}
$\mathrm{Ag} / \mathrm{Bi} 2223$ tapes were fabricated using the Powder-In-Tube method, involving intermediate deformation techniques of sandwich rolling (SR), pressing (P) and normal rolling (NR). Magnetic field $B$ dependence of $J_{c}$ was measured. Depending on the relationship between $J_{c}$ and $B$, the irreversible magnetic field $B_{i r r}$ was determined and pinning force density $F$ was calculated. The experimental results showed that self-field- $J_{c}$ was linear with the relative density for SR-, P- and NR-tapes. Our experimental results support that NR-, P-, and SR-tapes have a same sort of pinning center and the intermediate deformation processing cannot change property of pinning centers, but can effect the pinning force strength. Our experimental results also support that sandwich rolling is the best technique for fabricating $\mathrm{Ag} / \mathrm{Bi}-2223$ tapes.
\end{abstract}

\section{INTRODUCTION}

$\mathrm{Ag} / \mathrm{Bi} 2223$ multi-filamentary tapes in the high temperature superconducting family were one of the most promising superconductors for applications such as high current cables and strong magnets. A large effort was done to improve the properties of the tapes. Powder-In-Tube method was normally utilised to fabricate the tapes, in which thermal and mechanical processes were altermate to be used. In fact, the quality of tapes was critically dependent on those thermal and mechanical processes. To study the dependence of different mechanical deformations on the current and magnetic properties of tapes, the process of two sintering treatments with an intermediate deformation was carried out. The intermediate deformation processing was known as one of important processing to improve the quality of tapes for applications on high currents at different magnetic field: Techniques of normal rolling (NR), pressing (P) and sandwich rolling (SR) were used in this experiment as intermediate deformations. NR was a traditional processing of the intermediate deformation [1-5]. P often used in laboratories was the second method to improve the tape properties, especially to increase the core density of tapes $[6,7]$. SR was a special deformation technique that a sample is held between two spring-steel strips (i.e, 'sandwiched'), and the 'sandwich' was rolled. Sk prucessing developed in the recent years was found to be a useful intermediate deformation method, but has not been thoroughly studied yet [8-10]. To compare the differences among the three deformations, we fabricated $\mathrm{Ag} / \mathrm{Bi}-2223$ tapes by using the above three methods.

CP614, Advances in Cryogenic Engineering: 


\section{EXPERIMENTS}

$\mathrm{Ag} / \mathrm{Bi} 2223$ multifilamentary tapes with the nominal stoichiometry of $\mathrm{Bi}_{1.83} \mathrm{~Pb}_{0.34} \mathrm{Sr}_{1.91} \mathrm{Ca}_{2.03} \mathrm{Cu}_{3.05} \mathrm{O}_{\mathrm{y}}$ were fabricated by the powder-in-tube method, involving two last sintering processes with an intermediate deformation $[4,8,11]$. For comparison of experimental results, all samples used here were cut from the same green tape with an identical length before two last sintering processes. First last sintering was at $840^{\circ} \mathrm{C}$ for 50 hours in air, and the second one at $840^{\circ} \mathrm{C}$ for 30 hours and annealed at $825^{\circ} \mathrm{C}$ for 30 hours in air, followed by a furnace-cooling to room temperature. Between the two sintering processes, an intermediate deformation of NR, $P$ and $S R$ was performed with reduction rate from $0 \%$ to about $35 \%$. The optimum reduction rates with the maximum $J_{c}$ of tapes appeared at $22 \%, 25 \%$ and $21 \%$ for SR-, P- and NR-tapes, respectively [8,9]. To compare the three deformation methods, the three samples with maximum $J_{c}$ for SR-, P- and NRtapes were chosen for pinning force study.

To obtain core density dependence of $J_{c}$, we estimated a relative core density of deformed tapes, which is relative to undeformed one. We firstly measured length, width and thickness of as-sintered samples, and then calculated the volumes of the tapes. Noting that all the original samples had same amount of core mass because they had a same initial length. Thus, the relative density of a deformed sample can be determined by dividing its reciprocal volume by the reciprocal volume of the undeformed sample.

It is noted that the magnetisation strength $M$ of high- $T_{\mathrm{c}}$ superconductors is much less than the applied magnetic field $H$. For the sake of convenience, we take magnetic induction intensity $\boldsymbol{B}$ instead of applied magnetic field $H$ in following text. The critical current was measured by the four-electrode method, with a criterion of $1 \mu \mathrm{V} / \mathrm{cm}$. Magnetic field dependence of $J_{c}$ was measured at $77 \mathrm{~K}$ and the magnetic field was applied parallel to and perpendicular to the broad surface of the tapes, denoted by $B / / a b$ and $B / / c$, respectively.

Scanning Electron Microscopy (SEM) was applied to observe the crystal morphology and the grain texture of the samples. Major phase of Bi-2223 was determined by X-ray diffraction (XRD) analysis. Analysis of SEM and XRD showed that the samples had typical structures of $\mathrm{Bi}-2223$ phase.

\section{EXPERIMENTAL RESULTS}

Figure 1 shows the dependence of $J_{c}$ on $B$ in the direction of $B / / a b$ or $B / / c(0$ to $1.2 T)$ for samples with optimum reduction rate. Circular, star and triangle symbols express $J_{c}$ of tapes fabricated by SR, $\mathrm{P}$, and $\mathrm{NR}$, and open and solid ones for $B / / a b$ and $B / / c$, respectively. Both $J_{c}$ with $B / / a b$ and $B / / c$ decrease when $B$ increases, but the former's slope is lower than the later's. This result is consistent with that reported previously [12].

On the other hand, curves of $J_{c}$, according to directions of applied magnetic field, can be divided into two groups: one with $J_{c}(B / / c)$ decreasing sharply with increasing $B$, and the other with $J_{c}(B / / a b)$ decreasing more slowly with increasing $B$. For each group, $J_{c}$ dependence on $\mathrm{B}$ is different for various deformation methods. With increasing $B, J_{c}$ of NR-tape decreases fastest, that of P-tape the second and SR the slowest for both $B / / c$ and $B / / a b$.

Pinning force density, $F$, is defined as a vector product: $F=J_{c} \times B$. In our experiments, vector $\boldsymbol{B}$ is always perpendicular to the direction of current $J_{\boldsymbol{c}}$, therefore, the vector product becomes a simple scalar product, i. e. $F=J_{c}^{\bullet} B$. Values of $J_{c}$ and $B$ were recorded from experiments and values of $F$ obtained by $J_{c}$ multiplying by $B$. Figure 2 


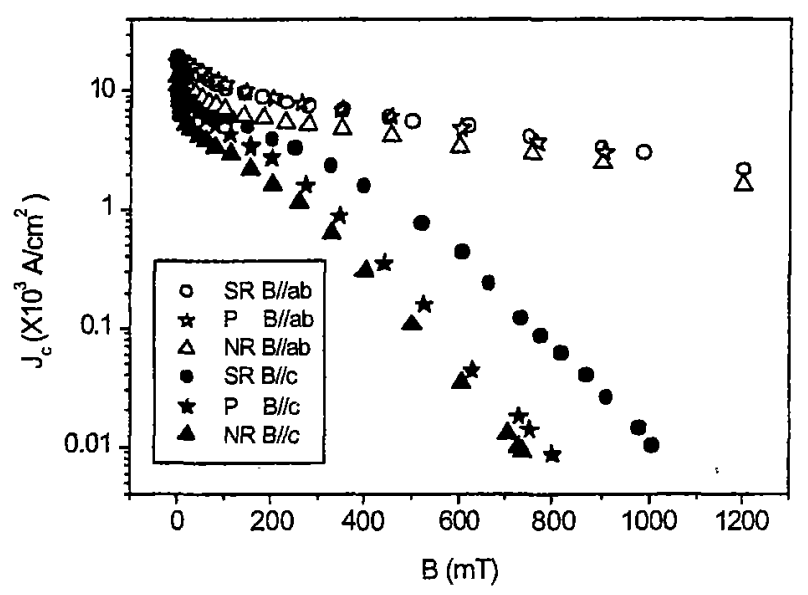

FIGURE 1. Critical current density $J_{c}$ dependence on magnetic field $B$ for tapes fabricated by SR, $\mathrm{P}$ and NR, respectively. The trends of curves, according to the direction of applied magnetic field, can be divided into two groups. Values of $J_{c}$ with $B / / a b$ are much larger than that with $B / / c$. For each group, SR tape has the highest $J_{c}, \mathrm{P}$ and NR tape the second and the third, respectively.

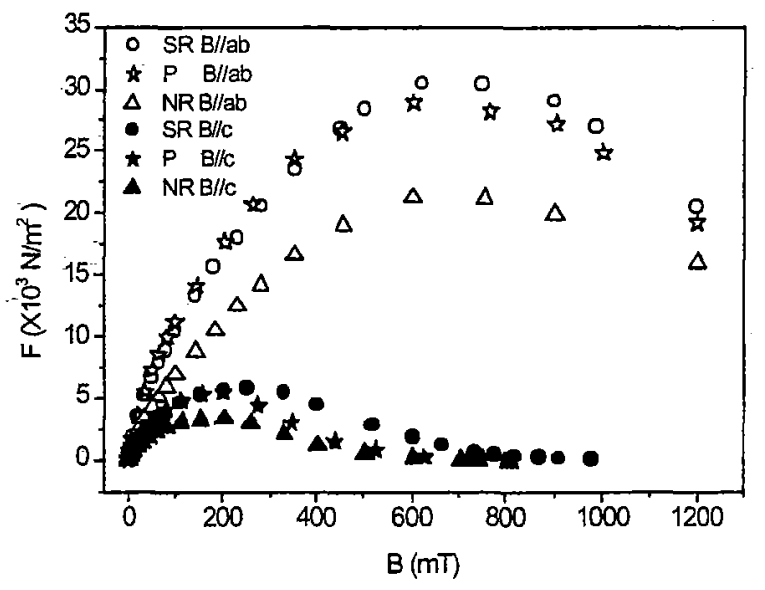

FIGURE 2. Variation of pinning force density $F$ on magnetic field $B$. The intensities of $F$ with $B / / a b$ are larger than that of $F$ with $B / / c$, and the former is about five times larger than latter for all three processings. The fact that curves in each case have similar trend means that tapes fabricated by SR, P and NR processing have same sort of pinning centers.

shows $B$ dependence of $F$, in which the upper three curves are for $B / / a b$, and the lower three for $B / / c$.

Irreversible magnetic field $B_{i r r}$ was determined by the following criterion: when applied magnetic field increases to a special value, $B_{i r}$, at which $J_{c}$ reduces to $1 \%$ of the zero magnetic field value, as shown in Fig. 3. For NR-, P- and SR- tapes, the values of $B_{i r}$ with $B / / c$ are listed in the Table I. 


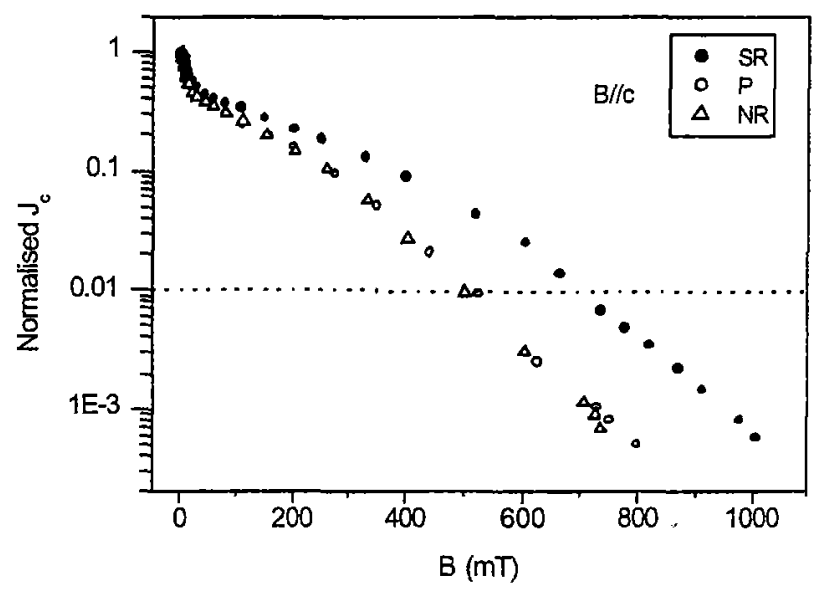

FIGURE 3. Dependence of normalised logarithmic $J_{c}$ of SR, P and NR tapes on magnetic field $B$. Irreversible magnetic field $B_{i r r}$ is determined from Y-axis label of 0.01 .

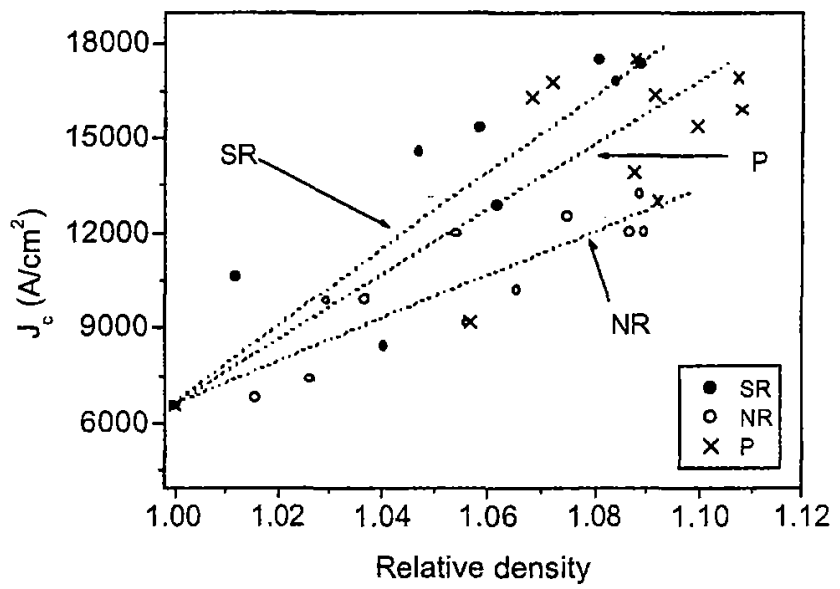

FIGURE 4. Self-field $J_{c}$ are proportional to its core density for tapes fabricated by SR, P, and NR techniques. The dot lines are the linear fitting lines. For different processing, the linear slope is different from each other, that of SR is the largest slope, that of $P$ and NR the second and third, respectively. It is found that at any core density, the SR tape has the largest $J_{c}$, the $\mathrm{P}$ the second, and the NR the third.

To study the core density dependence of $J_{c}$, we measured the self-field critical current of tapes with a series of reduction rates. Figure 4 shows the core density dependence of self- field $J_{c}$ for NR-, P- and SR- tapes. The scattering in the data of $J_{c}$ vs relative density is largely due to the uncertainty in the tape cores density measurement, but the linear tread of the data for each deformation technique is clearly. 
TABLE I. Maximum values of pinning force density, $F_{\text {max }}$, and irreversibility magnetic field, $B_{F \max }$, for $\mathrm{SR}$ $P$ and NR tapes, determined from Figures 2 and 3.

\begin{tabular}{|c|c|c|c|c|c|}
\hline $\begin{array}{c}\text { Physics Quantities } \\
\text { (units) }\end{array}$ & $\begin{array}{c}F_{\max } \\
\left(10^{3} \mathrm{~N} / \mathrm{m}^{2}\right)\end{array}$ & $\begin{array}{c}F_{\max } \\
\left(10^{3} \mathrm{~N} / \mathrm{m}^{2}\right)\end{array}$ & $\begin{array}{c}B_{\text {Fmax }} \\
(m T)\end{array}$ & $\begin{array}{c}B_{\text {Fmax }} \\
(m T)\end{array}$ & $\begin{array}{c}B_{\text {irr }} \\
(m T)\end{array}$ \\
\hline Magnetic field & $B / / a b$ & $B / / c$ & $B / / a b$ & $B / / c$ & $B / / c$ \\
\hline SR & 30.7 & 5.85 & 250 & 680 & 690 \\
\hline P & 28.9 & 5.58 & 195 & 627 & 528 \\
\hline NR & 22.4 & 3.50 & 152 & 623 & 500 \\
\hline
\end{tabular}

\section{DISCUSSION}

\subsection{Deformation processing dependence of $J_{c}$}

Figure 1 shows that $J_{c}$ curves of R-, P- and SR- tapes with $B / / a b$ direction are quite similar, but their magnitudes are different for different reduction techniques. The SR- tape has the largest $J_{c}$, the P and NR -tapes are the second and the third, respectively. However, $J_{c}$ on the $B / / c$ direction shows differences for NR, P and SR-tapes. $J_{c}$ of SR-tape decreases more slowly than that of $\mathrm{P}$ - and NR-tapes with increasing $B$. The experimental results: support that tape made by 'sandwich' rolling technique has the highest $J_{c}$ compared to those by pressing and normal rolling methods.

\subsection{Magnetic field dependence of $F$}

In fact, the behaviour of $J_{c}$ can be studied from the view of pinning force. Figure 2 shows $B(B / / a b$ and $B / / c)$ dependence of $F$ for NR-, P- and SR-tapes, respectively. Depending on magnitudes of $F$, curves of $F$ with applied magnetic direction can be divided in two groups, i.e. F with $B / / a b$ and $B / / c$. The trends of $F$ are only dependent on the direction of $B$ and independent on intermediate deformation techniques. The ratio between $F_{\max }$ with $B / / a b$ and that with $B / / c$ is about 5 for each deformation technique, i.e., $F_{\max }$ $(B / / a b) \cong 5 F_{\max }(B / / c)$. The similarity of $F-B$ curves as shown in Fig. 2 suggests that tapes processed by the three deformation techniques have same sort of pinning centres. For $B / / a b$, SR and $\mathrm{P}$ tapes almost have the same magnitude of $F$, which is much larger than that for NR tape. It is noted that core densities of filaments are different. $\mathrm{P}$ tape has the highest density; SR tapes the second and NR the third. $F$ is the pinning force for one unit area.

But for different deformation technique, the quantity and quality of pinning centres are different. Magnitude of maximal pinning force density $F_{\max }$ represents the strength of pinning force; both $F_{\max }$ and magnetic field $B_{F_{\max }}$ (at which the $F_{\max }$ appears) show properties of pinning centers on the magnetic field. The values of $F_{\max }$ and $B_{F \max }$ are different for the three reduction techniques. The tape made by sandwich rolling has the largest values of $F_{\max }$ and $B_{F \max }$, and tapes by $\mathrm{P}$ and $\mathrm{NR}$ has the second and the third, respectively. And values of $F_{\max }$ and $B_{F \max }$ are listed in Table I. 


\subsection{Irreversibility magnetic field $B_{i r r}$}

Irreversibility magnetic field $B_{i r r}$ is another basic physical quantity to characterise property of supercond uctors on high magnetic field. As is known, at critical magnetic field $H_{c 2}$, superconducting state changes into normal state. On the view of superconductivity, $H_{c 2}$ is the significant quantity to describe the superconducting property of a superconductor on high magnetic field. But, $H_{c 2}$ is too high to be measured for high $T_{c}$ superconductors, and, therefore, it cannot directly be used in practices. For applications, $B_{\text {irr }}$ is introduced, although the value of $B_{i r r}$ is different due to different definition. We take the criterion of $1 \%$ of $J_{c}$ at which magnetic field is recorded as $B_{u r r}$. Our experimental results show that deformation techniques affect the values of $B_{i r r}$. Table I shows that SR tape has the largest, the $\mathrm{P}$ the second and NR the third. SR processing also shows its advantages on $B_{i r}$.

\section{$4.4 J_{c}$ depending on relative core density}

Figure 4 shows self-field $J_{c}$ dependence on relative core density of tapes fabricated by $\mathrm{SR}, \mathrm{P}$ and $\mathrm{NR}$, respectively. The relationship between $J_{c}$ and core density for each deformation technique is approximately linear and the slope of the linearity varies with deformation techniques. Values of slopes are low, and that means that $J_{c}$ are strongly dependent on core density. Deformation techniques also affect the value of slopes. The SR processing has the largest slope, the $\mathrm{P}$ and NR the second and third, respectively. Therefore, SR processing is the best method to increase $J_{c}$ of tape.

\section{CONCLUSION}

- NR-, P-, and SR-tapes have same sort of pinning centers due to their similar relationships of $J_{c}$ vs $B$ and $F$ vs $B$.

- The evidence that tapes made by different intermediate deformation techniques have different strength of pinning force suggests that the intermediate deformation processing cannot change property of pinning centers, but can affect the pinning force strength.

- Our experimental results also show that tape fabricated by SR has the strongest pinning centers, that $P$ the second, and NR the third. All evidences presented above support that 'sandwich' rolling processing is the best intermediate reduction technique for fabricating $\mathrm{Ag} / \mathrm{Bi} 2223$ tapes.

\section{ACKNOWLEDGEMENTS}

This work is financially supported by Australian Research Council Large Grant.

\section{REFERENCES}

1. Liu, Y. L., Wang, W. G., Poulsen, H. F., and Supercond, P. Vase. Sci. Technol., 12, pp. 376 (1999).

2. Li, Q., Riley Jr, G. N., Parrella, R. D., Fleshler, S., Rupich, M. W., Carter, W. L., Willis, J. Q., Bingert, J. F., Sika, V. K., Parrell, J. A., and Larbalestier, D. C., IEEE trans. On Appl. Superconductivity 7, No 2, pp. 2026 (1997).

3. Kovac, P., Richens, P. E., Bukva, P., Jones, H., and Husek, I., Supercond. Sci. Technol,, 12, pp. 168 (1999). 
4. Merchant, N. N., Luo, J. S., Fischer, A. K., Maroni, V. A., Podtburg, E. R., Carter, W. L., Li, Q., A., Otto, M., Rupich, W. and Riley Jr, G. N., Supercond. Sci. Technol., 12, pp. 327 (1999).

5. Anderson, J. W., Parrell, J. A., Sastry, P. V. P. S. S., and Larbalestier, D. C., IEEE trans. On Appl. Superconductivity 7, No 2, pp. 1422 (1997).

6. Han, Z., Skov-Hansen, P. and Freltoft, T., Supercond. Sci. Technol., 10, pp. 371 (1997).

7. Zhu, Y. T., Baldonado, P. S., Bingert, J. F., Holesinger, T. G., Willis, J. O. and Peterson, D. E., Supercond. Sci. Technol., 12, pp. 540 (1999).

8. Chen, W. M., Guo, Y. C., Fu, X. K., Liu, H. K., Dou, S. X. and Apperley, M., IEEE trans. On Appl. Supercond. 11, No2, pp. 3752 (2001).

9. Chen, W.M., Guo, Y.C., McCaughey, G., Apperley, M., Liu, H.K. and Dou, S.X., Physica C 354, $349(2001)$.

10. Wang, W. G., Liu, H. K., Guo, Y. C., Bain, P. and Dou, S. X., Applied Superconductivity 3, No 11/12 pp. 599 (1995).

11. Marti, F., Grasso, G., Huang, Y., and Fluekiger, R., IEEE trans. On Appl. Superconductivity 7, No 2, pp. 2215 (1997).

12. W. M. Chen, F. Wang, F. Lin, S. S. Jiang, Y. C. Guo, H. K. Liu and S. X. Dou, Advances in Cryo. Eng: (Materials), 46, pp. 519(2000). 